\title{
The EST project
}

\author{
Francesca Zuccarello ${ }^{1,2}$ and the EST team \\ ${ }^{1}$ Dipartimento di Fisica e Astronomia - Universitá di Catania, Via S. Sofia 78, \\ I-95123 Catania, Italy \\ ${ }^{2}$ Istituto Nazionale di Astrofisica - Osservatorio Astrofisico di Catania, Via S. Sofia 78, I-95123 \\ Catania, Italy \\ email: fzu@oact.inaf.it
}

\begin{abstract}
EST European Solar Telescope is a pan-european project, presently in its Conceptual Design Study financed by the European Commission in the framework of FP7, involving 29 partners, from 14 different countries. The EST project is aimed at the realization of a 4 -m class telescope, characterized by an optical design and a set of instruments optimized for extremely high resolution imaging and spectropolarimetric observations from near UV to NIR. EST will be four times larger than any existing high resolution solar telescope and it is designated with the highest priority among the ground-based, medium term (2016-2020) new projects in the ASTRONET Roadmap (Panel C). The EST instruments will measure fundamental astrophysical processes at their intrinsic scales in the Sun's atmosphere to establish the mechanism of magnetic field generation and removal, and of energy transfer from the surface to the upper solar atmosphere and eventually to the whole heliosphere. The conceptual Design Study started on February 2008 and will finish during 2011. EST will be operational at the same time as major ESA and NASA space missions aimed at studying solar activity.
\end{abstract}

Keywords. Instrumentation: high angular resolution, Sun: photosphere, Sun: chromosphere, Sun: magnetic fields

\section{Introduction}

The comprehension of physical processes occurring in solar phenomena has been greatly improved in the past decades thanks to space-borne satellites (YOHKOH, SOHO, TRACE, RHESSI, HINODE) and to high resolution ground-based telescopes (THEMIS, VTT, SST, DOT, DST). Nevertheless, despite the important progresses achieved, there are still several aspects that need to be further investigated by means of higher temporal, spatial and spectral resolution data (Title 2010).

Moreover, if we take into account that some mechanisms occurring on the Sun are often considered to take place in other astrophysical contexts, it is becoming increasingly clear that the necessity of new-generation solar telescopes, of the 4-m class, can be shared, besides than by solar physicists, also by researchers involved in the study of plasma physics, Earth-magnetosphere, stellar activity, binary systems (see, e.g., Zhang 2007), accretion disks around black holes (Yuan et al. 2009), and other topics.

On the basis of these needs, new-generation solar telescopes have been promoted in the last years both in the USA and in Europe: the Advanced Technology Solar Telescope (ATST) is now at the beginning of the construction phase, while the European Solar Telescope (EST) is currently in the conclusive phase of the Design Phase.

In particular, the EST project is promoted by the european solar physics community by means of EAST (European Association for Solar telescopes), a consortium formed by research organizations from 15 European countries (Austria, Croatia, Czech Republic, France, Germany, Great Britain, Hungary, Italy, The Netherlands, Norway, Poland, 
Slovakia, Spain, Sweden and Switzerland), aimed at the development, realization and management of the telescope.

The Conceptual Design Phase of EST, financed by the European Commission in the framework of FP7 - Capacities Specific Programme - Research Infrastructures, is lead by the Institute de Astrofísica de Canarias, and is developed by 29 european partners (14 research institutions and 15 companies).

\section{The Current Design}

The current baseline design of EST is based on a 4-meter class solar telescope characterized by an on-axis Gregorian configuration, which will allow to achieve a good polarimetric performance. Three different types of instruments will be located in a controlled laboratory at the Coudé focus: a Broad Band Imager, a Narrow-Band Tunable Filter Spectropolarimeter and a Grating Spectropolarimeter (Collados et al. 2010). Each instrument will have several channels in order to observe simultaneously at various wavelengths.

The telescope mechanical configuration is alt-azimuthal in order to obtain, compared to the equatorial configuration, a more compact system and a better air flushing on the primary mirror, besides than allowing to achieve a polarimetric compensated optical design with less optical surfaces.

The telescope design includes adaptive optics (AO) and multi-conjugate adaptive optics (MCAO), consisting of deformable mirrors at conjugated heights, able to correct for the diurnal variation of the Sun's distance to the turbulence layers. The MCAO are integrated in the telescope optical path, allowing to maximize the telescope throughput and to provide simultaneously a corrected image at the Coudé focus for the three instruments. It is also foreseen that the MCAO train would be by-passable.

In order to allow the user to find the desired target on the solar disk, an auxiliary full-disc telescope (AFDT, Klvana et al., 2008) is foreseen. The AFDT has an aperture of $150 \mathrm{~mm}$ and can operate simultaneously in three spectral regions: Ca II K (394 nm), $\mathrm{H} \alpha(656.3 \mathrm{~nm})$, and white light (450 - $460 \mathrm{~nm})$.

Two different options have been considered for the enclosure: a completely retractable enclosure and a conventional dome. To date, the analysis carried out indicates that the foldable enclosure can provide better local seeing conditions and would allow the use of a reflecting heat rejecter at the Gregorian focus, but on the other hand it can produce higher wind effect on image quality. The conventional dome would allow a lower effect of wind on image quality, but the local seeing degradation might be larger and it would be necessary to control the environmental conditions inside the dome.

\subsection{The Telescope Optical Design}

The current baseline optical layout consists of 14 polarimetrically compensated mirrors. The elevation axis (line joining M4 and M5, see Fig. 1 (a)) is placed $1.5 \mathrm{~m}$ below the vertex of the primary mirror: this configuration facilitates M1 air flushing and allows space enough for the M1 cell and for an adequate placement of the transfer optics train vertically from the telescope to the Coudé focus. Moreover, the elevation and azimuth (line joining M7 and M8) axes are decentered with respect to the telescope optical axis so that the optical path is fold in an asymmetric way to produce a polarimetric compensated layout. In the F1 focus generated by the primary mirror M1, a heat-stop will be located, in order to remove most of the solar light and to allow a field-of-view of $(2-3) \times(2$ 3) $\operatorname{arcmin}^{2}$. In focus F2, generated by the secondary mirror, M2, the calibration optics will be installed to analyze the polarimetric performance of the rest of the telescope, and 


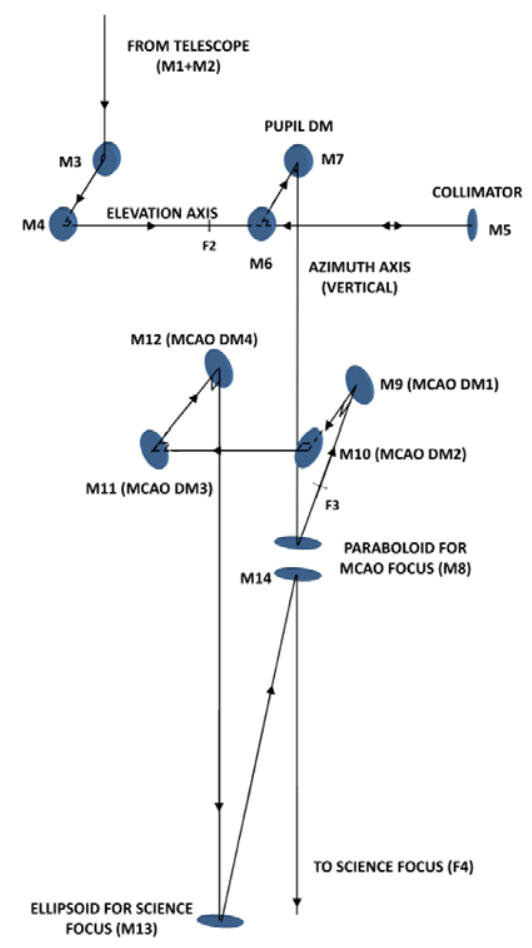

(a)

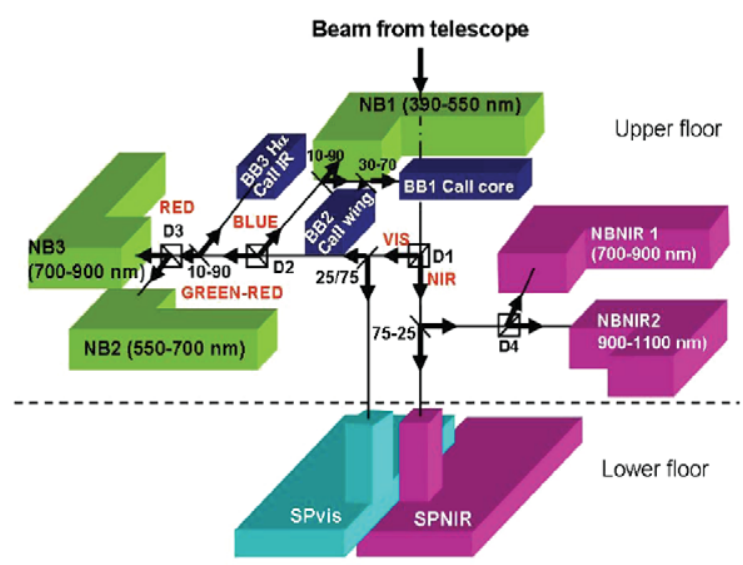

(b)

Figure 1. (a) Schematic drawing of the optical elements of the telescope. Mirrors M3 and M4 (as well as M6 and M7) are polarimetrically compensated. Mirrors M9 to M12 are the MCAO mirrors. (b) Schematic drawing of the instrument set-up designed for the EST telescope (from Cavaller et al. (2010)).

possibly also polarimetric modulators for polarization measurements. M5 is a collimator that produces an image of the pupil where a deformable mirror, DM (indicated by M7 in Fig. 1 (a)), is located to correct for the ground-layer turbulence. The flat mirror M6 will have tip-tilt correction capabilities to avoid image motion.

The MCAO mirrors are located after F3, the focus generated by M8: the present design has four MCAO DMs, conjugated at 5, 9, 15 and $30 \mathrm{~km}$, which operate at 45 degrees in a polarimetric compensated configuration. M13 generates the science focal plane, F4.

The present optical design adds three additional flat mirrors to this basic layout. The first two define the elevation axis of the telescope, and are placed in such a way that their incidence-reflection planes are perpendicular one to the other (mirrors M3 and M4 in Fig. 1 (a)), so that their instrumental polarization is cancelled. Furthermore, M6 and M7 (tip-tilt mirror and pupil DM, respectively), arranged in the same geometrical configuration as M3 and M4, are also polarimetrically compensated. As the elevation axis is defined by the line joining M4 and M5 and the azimuth axis by the line joining M7 and M8, the polarization of the incoming light is not modified, independently of the pointing of the telescope to any direction on the sky. If we consider the transfer optics, which includes the MCAO system, the four high-altitude DMs are also distributed to compensate their polarization properties. M14 is introduced to send the light to the Coudé room.

The transfer optics can be used as an optical de-rotator if the input optical axis (line joining the centers of M7 and M8) and the output optical axis (line joining M14 and F4) 
coincide (see Fig. 1 (a)). This avoids the inclusion of any derotating device at instrument level, such as a rotating platform, so that instruments can be kept fixed.

\subsection{The Instruments}

The instruments, composed of different channels at the Coudé focus, will be placed in a laboratory with a controlled environment, distributed in two floors (see Fig. 1 (b)). A system composed of dichroic and intensity beam-splitters will be used to feed the different instruments channels, in order to allow simultaneous observations.

In the current design the light is first split by a dichroic beam-splitter D1 in a visible and a near-infrared beam (see Fig. 1 (b)). Dichroic beam-splitters D2 and D3 will split further the visible beam in three narrower band-passes $(390-550 \mathrm{~nm}, 550-700 \mathrm{~nm}$, and $700-900 \mathrm{~nm}$ ), where the corresponding broad-band and narrowband channels are placed. Intensity beam-splitters (inclined lines crossing the beam in Fig. 1 (b)), are then used to feed all the instrument channels. The same conceptual design is used for the near-infrared branch. The Broad-Band Imager has two different operational modes: (a) high resolution mode $\left(1 \times 1 \operatorname{arcmin}^{2}, 0.015 \mathrm{arcsec} / \mathrm{pix}, 4 \mathrm{k} \times 4 \mathrm{k}\right.$ detectors $)$; (b) large field of view mode $\left(2 \times 2 \operatorname{arcmin}^{2}, 0.03 \mathrm{arcsec} / \mathrm{pix}, 4 \mathrm{k} \times 4 \mathrm{k}\right.$ detectors $)$.

The Narrow-Band, tunable filter imaging system, is based on Fabry-Pérot interferometers. The etalons could be mounted within the optical light beam either in the collimated configuration, or in the telecentric configuration.

Four different alternatives have been evaluated for the Grating Spectropolarimeters (each having a visible and a near-IR configuration): i) Long-Slit Standard Spectrograph; ii) Multi-Slit Multi-Wavelength Spectrograph; iii) Tunable Universal Narrow-band Imaging Spectrograph (TUNIS; López Ariste et al., 2010); iv) New generation Multi-channel Subtractive Double Pass (MSDP).

\section{Conclusions}

The EST Design phase will finish during the year 2011: at this time several decisions will be taken on the different alternatives that have been proposed so far. The site seeing campaign will also allow to take a decision on the site in the Canary Islands where the telescope will be built. EST construction is expected to start in 2014 and first light is foreseen in 2019 .

\section{Acknowledgements}

The conceptual design of EST is partially supported by the European Commission through the collaborative project Nr. 212482 EST: the large aperture European Solar Telescope under framework programme FP7.

\section{References}

Cavaller L., et al. 2010, "EST: European Solar Telescope. Design study phase II report", EST project documentation, report RPT-GTC

Collados, M.; Bettonvil, F.; Cavaller, L.; Ermolli, I.; Gelly, B.; Pérez, A.; Socas-Navarro, H.; Soltau, D.; Volkmer, R.; EST team 2010, AN, 331, 615

Klvana, M., Sobotka, M., Svanda, M. 2008, in: 12th European Solar Physics Meeting, Online at http://espm.kis.unifreiburg. de/, p. 2.73

López Ariste, A., LeMen, C., Gelly, B., Asensio Ramos, A. 2010, AN 331, 658

Title, A. 2010, $A N, 331,599$

Yuan, F., Lin, J., Wu, K., \& Ho, L. C. 2009, Mon. Not. R. Astron. Soc., 395, 2183

Zhang, S. N. 2006, Highlights of Astronomy, 14, 119 